\title{
On the sunflower bound for $k$-spaces, pairwise intersecting in a point
}

\author{
A. Blokhuis*, M. De Boeck ${ }^{\dagger}$ J. D’haeseleer ${ }^{\ddagger}$
}

\begin{abstract}
A $t$-intersecting constant dimension subspace code $C$ is a set of $k$ dimensional subspaces in a projective space $\operatorname{PG}(n, q)$, where distinct subspaces intersect in exactly a $t$-dimensional subspace. A classical example of such a code is the sunflower, where all subspaces pass through the same $t$-space. The sunflower bound states that such a code is a sunflower if $|C|>\left(\frac{q^{k+1}-q^{t+1}}{q-1}\right)^{2}+\left(\frac{q^{k+1}-q^{t+1}}{q-1}\right)+1$.

In this article we will look at the case $t=0$ and we will improve this bound for $q \geq 9$ : a set $\mathcal{S}$ of $k$-spaces in $\operatorname{PG}(n, q), q \geq 9$, pairwise intersecting in a point is a sunflower if $|\mathcal{S}|>\left(\frac{2}{\sqrt[6]{q}}+\frac{4}{\sqrt[3]{q}}-\frac{5}{\sqrt{q}}\right)\left(\frac{q^{k+1}-1}{q-1}\right)^{2}$.
\end{abstract}

Keywords: Subspace codes, $q$-analogue problems, Sunflower bound, Random network coding.

MSC 2010 codes: 51E20, 05B25, 52E14, 51E23, 51E30.

\section{Introduction}

In a vector space $V$, a $(k, t)$-SCID is a set of $k$-dimensional subspaces of $V$, pairwise intersecting in exactly a $t$-dimensional subspace (SCID stands for: $S u b$ spaces with Constant Intersection Dimension). We will work in a projective context, so a $(k+1, t+1)$-SCID corresponds to a set of $k$-dimensional projective subspaces in $\mathrm{PG}(n, q)$, that pairwise intersect in a $t$-dimensional space (see [4]). A $(k+1, t+1)$-SCID is also called a $t$-intersecting constant dimension subspace code, where the code words have projective dimension $k$. Note that $(k+1,0)$-SCIDs correspond with partial $k$-spreads in $\mathrm{PG}(n, q)$.

An example of a $(k+1, t+1)$-SCID is a sunflower, which is a set of $k$-spaces, passing through the same $t$-space and having no points in common outside this $t$-space. It can be shown that a $t$-intersecting constant dimension subspace code is a sunflower if the code has many code words.

\footnotetext{
* Department of Mathematics and Computer Science, Eindhoven University of Technology, The Netherlands, a.blokhuis@tue.nl.

${ }^{\dagger}$ Department of Mathematics: Algebra and Geometry, Ghent University, Flanders, Belgium, maarten.deboeck@ugent.be.

$¥$ (corresponding author) Department of Mathematics: Analysis, Logic and Discrete Mathematics, Ghent University, Flanders, Belgium, jozefien.dhaeseleer@ugent.be.
} 
Theorem 1.1. [5, Theorem 1] $A(k+1, t+1)$-SCID $C$ is a sunflower if

$$
|C|>\left(\frac{q^{k+1}-q^{t+1}}{q-1}\right)^{2}+\left(\frac{q^{k+1}-q^{t+1}}{q-1}\right)+1 .
$$

It is believed that the sunflower bound is in general not tight. In [2], the sunflower bound for $(k+1,1)$-SCIDs was studied.

Theorem 1.2 ([2, Theorem 2.1]). Let $C$ be $a(k+1,1)-S C I D$, with $k \geq 4$. If

$$
|C| \geq\left(\frac{q^{k+1}-q}{q-1}\right)^{2}+\left(\frac{q^{k+1}-q}{q-1}\right)-q^{k}
$$

then $C$ is a sunflower.

In this article we will give a better result for $(k+1,1)$-SCIDs. In [1] a geometrical sunflower bound was studied: the authors investigate SCIDs that span a large subspace. They prove that sunflowers are the SCIDs that span the 'largest' subspaces.

We suppose that $k \geq 3$ as for $(2,1)$-SCIDs we, more generally, known that every $(k+1, k)$-SCID is a sunflower or consists of all $k$-spaces in a fixed $(k+1)$ space. For $(3,1)$-SCIDs, an almost complete classification is known, see [3].

In Section 2, we give some definitions and general lemmas. In Section 3, we start with the Main Lemma that gives an important inequality. Using this inequality we continue with Theorem 3.6 that gives an improvement on the sunflower bound if $k \geq 3$ and $q \geq 9$ (and if $q \geq 7$ and $k \geq 5$ ).

\section{Preliminaries}

We start with the definition of the q-ary Gaussian coefficient.

Definition 2.1. Let $q$ be a prime power, let $n, k$ be non negative integers with $k \leq n$. The q-ary Gaussian coefficient of $n$ and $k$ is defined by

$$
\left[\begin{array}{l}
n \\
k
\end{array}\right]_{q}=\left\{\begin{array}{cl}
\frac{\left(q^{n}-1\right) \cdots\left(q^{n-k+1}-1\right)}{\left(q^{k}-1\right) \cdots(q-1)} & \text { if } k>0 \\
1 & \text { otherwise }
\end{array}\right.
$$

We will write $\left[\begin{array}{l}n \\ k\end{array}\right]$, if the field size $q$ is clear from the context. The number of $k$-spaces in $\operatorname{PG}(n, q)$ is $\left[\begin{array}{c}n+1 \\ k+1\end{array}\right]$ and the number of $k$-spaces through a fixed $t$-space in $\operatorname{PG}(n, q)$, with $0 \leq t \leq k$, is $\left[\begin{array}{c}n-t \\ k-t\end{array}\right]$. Moreover, we will denote the number $\left[\begin{array}{c}n+1 \\ 1\end{array}\right]$ by the symbol $\theta_{n}$.

From now on we consider a fixed $(k+1,1)$-SCID $\mathcal{S}$ that is not a sunflower, of size $|\mathcal{S}|=(1-s) \theta_{k}^{2}, 0<s<1$. Note that the size of $|\mathcal{S}|$ is smaller than the sunflower bound for $s>\frac{1}{\theta_{k}}-\frac{1}{\theta_{k}^{2}}$. We will give, for a fixed value of $k$ and field size $q$, an upper bound on $1-s$. For convenience we will call a $k$-space contained in $\mathcal{S}$ a block. 
Definition 2.2. Consider the above set $\mathcal{S}$ of $k$-spaces, or blocks. The sets of points and lines that are contained in a block are denoted by $\mathcal{P}_{\mathcal{S}}$ and $\mathcal{L}_{\mathcal{S}}$ respectively.

Lemma 2.3. Suppose $P \in \mathcal{P}_{\mathcal{S}}$, then $P$ lies in at most $\theta_{k}$ blocks and on at most $\theta_{k} \cdot \theta_{k-1}$ lines of $\mathcal{L}_{\mathcal{S}}$.

Proof. Consider a block $S_{0}$ not through $P$ (such an $S_{0}$ exists since $\mathcal{S}$ is not a sunflower). Every block through $P$ contains a point $Q$ of $S_{0}$ and every line $P Q$ with $Q \in S_{0}$ is contained in at most one block. In this way we find at most $\theta_{k}$ blocks that contain $P$. The lemma follows since the number of lines through a point in a $k$-space is $\theta_{k-1}$.

From now on we distinguish 'rich' and 'poor' points and lines in $\mathcal{P}_{\mathcal{S}}$ and $\mathcal{L}_{\mathcal{S}}$. First we give the definition, then we continue with some counting arguments.

Definition 2.4. Suppose $c, d$ are constants between $s$ and 1 .

$A$ point $P \in \mathcal{P}_{\mathcal{S}}$ is $c$-rich if it is included in more than $(1-c) \theta_{k}$ blocks. A point is c-poor if it is not c-rich.

A line $l \in \mathcal{L}_{\mathcal{S}}$ is $(c, d)$-rich if it contains more than $(1-d)(q+1)$ c-rich points.

We will call $c$-rich and $c$-poor points, and $(c, d)$-rich lines rich and poor points, and rich lines respectively, if the constants $c$ and $d$ are clear from the context.

Lemma 2.5. For the number $r$ of c-rich points in a block, we find

$$
r \geq r_{0}=\left(1-\frac{s}{c}\right) \theta_{k} .
$$

Proof. Fix a block $S_{0}$ in $\mathcal{S}$, and count the number of elements in $\mathcal{S}$ that intersect $S_{0}$ in a point: through every rich point $P$ of $S_{0}$ there are at most $\theta_{k}-1$ blocks different from $S_{0}$, by Lemma 2.3. Through every line spanned by $P$ and a point of such a block, there is at most one block.

Every poor point of $S_{0}$ lies in at most $(1-c) \theta_{k}-1$ other blocks by definition. We double-count point-block pairs $(P, S)$ with $P \in S$ where $P \in S_{0}$ and $S \neq S_{0}$ to obtain the following inequality:

$$
\begin{array}{rlrl}
r\left(\theta_{k}-1\right)+\left(\theta_{k}-r\right)\left((1-c) \theta_{k}-1\right) & \geq|\mathcal{S}|-1 \\
\Leftrightarrow & & r\left(\theta_{k}-1-(1-c) \theta_{k}+1\right) & \geq(1-s) \theta_{k}^{2}-1-(1-c) \theta_{k}^{2}+\theta_{k} \\
\Rightarrow & & r c \theta_{k} & \geq(c-s) \theta_{k}^{2} \\
\Leftrightarrow & & r & \geq\left(1-\frac{s}{c}\right) \theta_{k} .
\end{array}
$$

Lemma 2.6. A block contains at least

$$
\frac{\theta_{k} \theta_{k-1}}{q+1} \cdot\left(1-\frac{s}{c d}\right)
$$

$(c, d)$-rich lines and the total number of $(c, d)$-rich lines is at least $(1-s) \theta_{k}^{2}$ times this number. 
Proof. Consider a block $S_{0} \in \mathcal{S}$ and let $\beta$ denote the number of poor lines in $S_{0}$. By counting pairs $(P, l)$, with $P$ a rich point in $S_{0}, l$ a line in $S_{0}$ and $P \in l$, we find:

$$
\left(\left[\begin{array}{c}
k+1 \\
2
\end{array}\right]-\beta\right)(q+1)+\beta(1-d)(q+1) \geq r_{0} \theta_{k-1}=\left(1-\frac{s}{c}\right) \theta_{k} \theta_{k-1},
$$

which gives

$$
\beta \leq \frac{s \theta_{k} \theta_{k-1}}{c d(q+1)}
$$

Hence, an element of $\mathcal{S}$ contains at least $\left[\begin{array}{c}k+1 \\ 2\end{array}\right]-\beta \geq \frac{\theta_{k} \theta_{k-1}}{q+1}-\frac{s \theta_{k} \theta_{k-1}}{c d(q+1)}$ elements.

Remark 2.7. In order to get a useful bound in the previous lemma, we need values of $s, c$ and $d$ such that $s \leq c d$. Later we will see that the values that we use for $c$ and $d$ satisfy these inequalities.

We continue with a lemma that will be useful to prove the Main Lemma and the theorems in the following section.

Lemma 2.8. The average number of $(c, d)$-rich lines meeting two distinct blocks $S_{1}, S_{2}$ in a c-rich point different from $S_{1} \cap S_{2}$ is at least

$$
f(s)=\theta_{k} \theta_{k-1} q \frac{1-d}{1-s}\left(1-\frac{s}{c d}\right)\left(1-c-\frac{1}{\theta_{k}}\right)^{2}\left(1-d-\frac{d}{q}\right) .
$$

Proof. We count triples $\left(S_{1}, S_{2}, r\right)$ where $r$ is a rich line connecting a rich point in $S_{1} \backslash S_{2}$ with a rich point in $S_{2} \backslash S_{1}$. Let $\rho_{\left\{S_{1}, S_{2}\right\}}, S_{1}, S_{2} \in \mathcal{S}, S_{1} \neq S_{2}$, be the number of rich lines meeting both $S_{1} \backslash S_{2}$ and $S_{2} \backslash S_{1}$ in a rich point. We define $\rho(s)$ as the average of the values $\rho_{\left\{S_{1}, S_{2}\right\}}$ with $S_{1}, S_{2} \in \mathcal{S}$ and $S_{1} \neq S_{2}$. On the one hand the number of triples equals

$$
(1-s) \theta_{k}^{2}\left((1-s) \theta_{k}^{2}-1\right) \rho(s) \leq(1-s)^{2} \theta_{k}^{4} \cdot \rho(s) .
$$

On the other hand, the number of triples is at least

$$
(1-s) \theta_{k}^{2} \frac{\theta_{k} \theta_{k-1}}{q+1}\left(1-\frac{s}{c d}\right) \cdot(1-d)(q+1) \cdot((1-d) q-d) \cdot\left((1-c) \theta_{k}-1\right)^{2}
$$

as by Lemma 2.6 , there are at least $(1-s) \theta_{k}^{2} \cdot \frac{\theta_{k} \theta_{k-1}}{q+1} \cdot\left(1-\frac{s}{c d}\right)$ rich lines, and on a rich line there are at least $(1-d)(q+1)((1-d) q-d)$ possibilities for an ordered pair of two distinct rich points $P_{1}, P_{2}$. Through those points we find at least $\left((1-c) \theta_{k}-1\right)^{2}$ possibilities for the blocks $S_{1}$ and $S_{2}$ (not containing the line $\left.P_{1} P_{2}\right)$. This gives that the average $\rho(s)$ is at least $f(s)$. 


\section{Main Lemma and results}

Using the combinatorial lemmas in the previous section, the main goal in this section is to find a an upper bound on $(1-s)$, as a function of the field size $q$. We start with the Main Lemma, that will be the basis of the theorems at the end of this section.

Main Lemma 3.1. Let $\mathcal{S}$ be a $(k+1,1)-S C I D$ in $\operatorname{PG}(n, q)$ with $|\mathcal{S}|=(1-s) \theta_{k}^{2}$, $k \geq 3$, that is not a sunflower. For all values $0<s<c, d<1$, we have the following inequality:

$$
\begin{array}{r}
\left(1-\frac{s}{c d}\right)(1-d)(1-c)\left(1-c-\frac{1}{q^{3}}\right)^{2}\left(1-d-\frac{d}{q}\right) \\
\left(1-d-\frac{1+d}{q}\right) q \\
\leq(1-s)^{2}+\frac{1-s}{q} .
\end{array}
$$

Proof. Consider a pair of different blocks $S_{1}, S_{2}$ such that there are at least $f(s)$ distinct $(c, d)$-rich lines connecting a point of $S_{1} \backslash S_{2}$ and a point of $S_{2} \backslash S_{1}$. Note that such a pair is guaranteed to exist by Lemma 2.8. Then, the $2 k$-space $T=\left\langle S_{1}, S_{2}\right\rangle$ contains at least

$$
f(s) \cdot \frac{(1-d)(q+1)-2}{q}=\left(1-d-\frac{1+d}{q}\right) f(s)
$$

rich points, since every point $P$ in the $2 k$-space $T$ not in the union $S_{1} \cup S_{2}$ lies on at most $q$ such connecting lines. Indeed, each such line is contained in the plane $\left\langle P, S_{1}\right\rangle \cap\left\langle P, S_{2}\right\rangle$, and in this plane there are $q$ lines through $P$ that do not contain $\mathcal{S}_{1} \cap \mathcal{S}_{2}$. Each of these $q$ lines might or might not be rich.

Since the dual of a $(k+1,1)$-SCID in a $2 k$-space is a partial $(k-1)$-spread in this $2 k$-space, we have that a $2 k$-space contains at most $\left\lfloor\theta_{2 k} / \theta_{k-1}\right\rfloor=q^{k+1}+q$ blocks. On the other hand, $T$ contains at most $\theta_{k-1}$ points from each block not contained in $T$. Hence the number of pairs $(P, S)$, with $P \in\left\langle S_{1}, S_{2}\right\rangle$ a rich point in the block $S$ is at least $(1-d-(1+d) / q) f(s)(1-c) \theta_{k}$ and at most $\left(q^{k+1}+q\right) \theta_{k}+\left((1-s) \theta_{k}^{2}-\left(q^{k+1}+q\right)\right) \theta_{k-1}$. Hence

$$
\begin{aligned}
& \left(1-d-\frac{1+d}{q}\right)(1-c) f(s) \theta_{k} \\
\leq & \left(q^{k+1}+q\right) \theta_{k}+\left((1-s) \theta_{k}^{2}-\left(q^{k+1}+q\right)\right) \theta_{k-1} \\
\Rightarrow \quad & \left(1-d-\frac{1+d}{q}\right)(1-c) \frac{f(s)}{\theta_{k} \theta_{k-1}} \leq 1-s+\frac{q^{k+1}+q}{\theta_{k}^{2} \theta_{k-1}} q^{k} \leq 1-s+\frac{1}{q^{k-2}} .
\end{aligned}
$$

The last inequality follows since $q^{k}\left(q^{k+1}+q\right) \leq q^{2-k} \theta_{k}^{2} \theta_{k-1}$. This implies that

$$
\begin{array}{r}
\left(1-\frac{s}{c d}\right)(1-d)(1-c)\left(1-c-\frac{1}{\theta_{k}}\right)^{2}\left(1-d-\frac{d}{q}\right) \\
\left(1-d-\frac{1+d}{q}\right) q \\
\leq(1-s)^{2}+\frac{1-s}{q^{k-2}}
\end{array}
$$


which proves the lemma since $k \geq 3$.

Corollary 3.2. Let $\mathcal{S}$ be a $(k+1,1)-S C I D$ in $\mathrm{PG}(n, q)$ with $|\mathcal{S}|=(1-s) \theta_{k}^{2}$, $k \geq 3$, that is not a sunflower. Suppose that

$$
\left(\frac{1}{q}-\frac{B(q, c, d)}{c d}\right)^{2}-4 B(q, c, d)\left(\frac{1}{c d}-1\right) \geq 0 .
$$

Then we have, for all values $0<s<c, d<1$, that

$$
\begin{aligned}
& (1-s) \leq F(q, c, d)=\frac{1}{2}\left(\frac{B(q, c, d)}{c d}-\frac{1}{q}-\sqrt{\left(\frac{1}{q}-\frac{B(q, c, d)}{c d}\right)^{2}-4 B(q, c, d)\left(\frac{1}{c d}-1\right)}\right) \\
& \text { or }(1-s) \geq G(q, c, d)=\frac{1}{2}\left(\frac{B(q, c, d)}{c d}-\frac{1}{q}+\sqrt{\left(\frac{1}{q}-\frac{B(q, c, d)}{c d}\right)^{2}-4 B(q, c, d)\left(\frac{1}{c d}-1\right)}\right) \\
& \text { with } B(q, c, d)=(1-d)(1-c)\left(1-c-\frac{1}{q^{3}}\right)^{2}\left(1-d-\frac{d}{q}\right)\left(1-d-\frac{1+d}{q}\right) q .
\end{aligned}
$$

Proof. Using inequality (1) from the Main Lemma, we immediately find the following quadratic inequality

$$
(1-s)^{2}+\left(\frac{1}{q}-\frac{B(q, c, d)}{c d}\right) \cdot(1-s)+B(q, c, d)\left(\frac{1}{c d}-1\right) \geq 0,
$$

which proves the corollary.

From now on, we put $c(q)=d(q)=1-\frac{1}{\sqrt[6]{q}}-\frac{1}{2 \sqrt[3]{q}}$. Since $c$ and $d$ must be non negative by definition, we have to assume that $q \geq 7$. We denote $c(q), F(q, c(q), c(q)), G(q, c(q), c(q))$ and $B(q, c(q), c(q))$ by $c_{q}, F_{q}, G_{q}$ and $B_{q}$ respectively. We first give two lower bounds on $B_{q}$.

Lemma 3.3. Let $t=\sqrt[6]{q}, q \geq 7$, then

$$
\begin{aligned}
& B_{q}>\left(1+\frac{1}{2 t}\right)^{2}\left(1+\frac{1}{2 t}-\frac{1}{t^{4}}\right)^{2}\left(1+\frac{1}{2 t}-\frac{1}{t^{5}}\right)\left(1+\frac{1}{2 t}-\frac{2}{t^{5}}\right) \\
& B_{q}>\left(1+\frac{1}{2 t}\right)^{2}\left(1+\frac{1}{3 t}\right)^{2} .
\end{aligned}
$$

Proof. By using the equality $c_{q}=c_{t^{6}}=1-\frac{1}{t}-\frac{1}{2 t^{2}}$ and $t \geq \sqrt[6]{7}$, we have

$$
\begin{aligned}
B_{q}= & \left(1-c_{q}\right)^{2}\left(1-c_{q}-\frac{1}{q^{3}}\right)^{2}\left(1-c_{q}-\frac{c_{q}}{q}\right)\left(1-c_{q}-\frac{1+c_{q}}{q}\right) q \\
= & \left(\frac{1}{t}+\frac{1}{2 t^{2}}\right)^{2}\left(\frac{1}{t}+\frac{1}{2 t^{2}}-\frac{1}{t^{18}}\right)^{2}\left(\frac{1}{t}+\frac{1}{2 t^{2}}-\frac{1}{t^{6}}+\frac{1}{t^{7}}+\frac{1}{2 t^{8}}\right) \\
& \left(\frac{1}{t}+\frac{1}{2 t^{2}}-\frac{2}{t^{6}}+\frac{1}{t^{7}}+\frac{1}{2 t^{8}}\right) t^{6} \\
= & \left(1+\frac{1}{2 t}\right)^{2}\left(1+\frac{1}{2 t}-\frac{1}{t^{17}}\right)^{2}\left(1+\frac{1}{2 t}-\frac{1}{t^{5}}+\frac{1}{t^{6}}+\frac{1}{2 t^{7}}\right) \\
& \left(1+\frac{1}{2 t}-\frac{2}{t^{5}}+\frac{1}{t^{6}}+\frac{1}{2 t^{7}}\right) .
\end{aligned}
$$


Using this expression for $B_{q}$, we can check that the following two inequalities are true for all $t \geq \sqrt[6]{7}$, and so, for all $q \geq 7$.

$$
\begin{aligned}
& B_{q}>\left(1+\frac{1}{2 t}\right)^{2}\left(1+\frac{1}{2 t}-\frac{1}{t^{4}}\right)^{2}\left(1+\frac{1}{2 t}-\frac{1}{t^{5}}\right)\left(1+\frac{1}{2 t}-\frac{2}{t^{5}}\right), \text { and } \\
& B_{q}>\left(1+\frac{1}{2 t}\right)^{2}\left(1+\frac{1}{3 t}\right)^{2} .
\end{aligned}
$$

We continue by investigating the condition $\left(\frac{1}{q}-\frac{B_{q}}{c_{q}^{2}}\right)^{2}-4 B_{q}\left(\frac{1}{c_{q}^{2}}-1\right) \geq 0$ from Corollary 3.2, proving for which values of $q \geq 7$ it is valid. Or equivalently, for which values of $q$, the argument of the square root in $F_{q}$ and $G_{q}$ is nonnegative.

Lemma 3.4. For $q \geq 7$ it is true that $\left(\frac{1}{q}-\frac{B_{q}}{c_{q}^{2}}\right)^{2}-4 B_{q}\left(\frac{1}{c_{q}^{2}}-1\right) \geq 0$, with $B_{q}=$ $\left(1-c_{q}\right)^{2}\left(1-c_{q}-\frac{1}{q^{3}}\right)^{2}\left(1-c_{q}-\frac{c_{q}}{q}\right)\left(1-c_{q}-\frac{1+c_{q}}{q}\right) q$ and $c_{q}=1-\frac{1}{\sqrt[6]{q}}-\frac{1}{2 \sqrt[3]{q}}$.

Proof. Note that it follows from Lemma 3.3 that $B_{q}>0$ if $q \geq 7$ (we will use this on the third line). Suppose that the inequality in the statement of the lemma does not hold. Then we have

$$
\begin{array}{ll} 
& \frac{B_{q}^{2}}{c_{q}^{4}}-\frac{2 B_{q}}{q c_{q}^{2}}+\frac{1}{q^{2}}<4 B_{q}\left(\frac{1}{c_{q}^{2}}-1\right) \\
\Rightarrow \quad & \frac{B_{q}^{2}}{c_{q}^{4}}<2 B_{q}\left(\frac{2}{c_{q}^{2}}-2+\frac{1}{q c_{q}^{2}}\right) \\
\stackrel{B_{q}>0}{\Longleftrightarrow} & B_{q}<2 c_{q}^{2}\left(2\left(1-c_{q}^{2}\right)+\frac{1}{q}\right) \\
\stackrel{t=\sqrt[6]{q}}{\Longleftrightarrow} & B_{t^{6}}<2\left(1-\frac{1}{t}-\frac{1}{2 t^{2}}\right)^{2}\left(\frac{4}{t}-\frac{2}{t^{3}}-\frac{1}{2 t^{4}}+\frac{1}{t^{6}}\right) \\
\stackrel{(4)}{\Longleftrightarrow} & \left(1+\frac{1}{2 t}\right)^{2}\left(1+\frac{1}{3 t}\right)^{2}<2\left(1-\frac{1}{t}\right)^{2}\left(\frac{4}{t}-\frac{1}{t^{3}}\right) \\
\Leftrightarrow \quad & \left(t+\frac{1}{2}\right)^{2}\left(t+\frac{1}{3}\right)^{2}<2(t-1)^{2}\left(4 t-\frac{1}{t}\right) \\
\Leftrightarrow \quad & t^{4}+\frac{5}{3} t^{3}+\frac{37}{36} t^{2}+\frac{5}{18} t+\frac{1}{36}<8 t^{3}-16 t^{2}+6 t+4-\frac{2}{t} \\
\Leftrightarrow \quad & t^{4}-\frac{19}{3} t^{3}+\frac{613}{36} t^{2}-\frac{103}{18} t-\frac{143}{36}+\frac{2}{t}<0 .
\end{array}
$$

The last inequality gives a contradiction for all values of $t \geq \sqrt[6]{7}$, and so for all $q \geq 7$, which proves the lemma.

Now we prove that $G_{q}>1$. This implies that the first bound in Corollary 3.2 holds, since $0<s<1$. 
Lemma 3.5. For $q \geq 7$, it is true that

$$
G_{q}=\frac{1}{2}\left(\frac{B_{q}}{c_{q}^{2}}-\frac{1}{q}+\sqrt{\left(\frac{1}{q}-\frac{B_{q}}{c_{q}^{2}}\right)^{2}-4 B_{q}\left(\frac{1}{c_{q}^{2}}-1\right)}\right)>1
$$

with

$$
\begin{aligned}
B_{q} & =\left(1-c_{q}\right)^{2}\left(1-c_{q}-\frac{1}{q^{3}}\right)^{2}\left(1-c_{q}-\frac{c_{q}}{q}\right)\left(1-c_{q}-\frac{1+c_{q}}{q}\right) q \text { and } \\
c_{q} & =1-\frac{1}{\sqrt[6]{q}}-\frac{1}{2 \sqrt[3]{q}} .
\end{aligned}
$$

Proof. We have to prove that

$$
\frac{B_{q}}{c_{q}^{2}}-\frac{1}{q}+\sqrt{\left(\frac{1}{q}-\frac{B_{q}}{c_{q}^{2}}\right)^{2}-4 B_{q}\left(\frac{1}{c_{q}^{2}}-1\right)}>2 .
$$

For all values of $q \geq 7$ such that $2-\frac{B_{q}}{c_{q}^{2}}+\frac{1}{q}<0$, the previous inequality is true. If $2-\frac{B_{q}}{c_{q}^{2}}+\frac{1}{q} \geq 0$, then it is equivalent to proving that

$$
\begin{aligned}
& \left(\frac{1}{q}-\frac{B_{q}}{c_{q}^{2}}\right)^{2}-4 B_{q}\left(\frac{1}{c_{q}^{2}}-1\right)>4+4\left(\frac{1}{q}-\frac{B_{q}}{c_{q}^{2}}\right)+\left(\frac{1}{q}-\frac{B_{q}}{c_{q}^{2}}\right)^{2} \\
\Leftrightarrow & -\frac{B_{q}}{c_{q}^{2}}+B_{q}>1+\frac{1}{q}-\frac{B_{q}}{c_{q}^{2}} \\
\Leftrightarrow & B_{q}>1+\frac{1}{q} .
\end{aligned}
$$

Set $t=\sqrt[6]{q}$. From Lemma 3.3(4), we know that it is sufficient to prove the following inequality:

$$
\begin{aligned}
& \left(1+\frac{1}{2 t}\right)^{2}\left(1+\frac{1}{3 t}\right)^{2}>1+\frac{1}{t^{6}} \\
\Leftrightarrow & t^{4}+\frac{5}{3} t^{3}+\frac{37}{36} t^{2}+\frac{5}{18} t+\frac{1}{36}>t^{4}+\frac{1}{t^{2}} \\
\Leftrightarrow & \frac{5}{3} t^{3}+\frac{37}{36} t^{2}+\frac{5}{18} t+\frac{1}{36}-\frac{1}{t^{2}}>0 .
\end{aligned}
$$

This last inequality is true for $t \geq \sqrt[6]{7}$, and so for $q \geq 7$, which proves the lemma.

Theorem 3.6. $A(k+1,1)$-SCID in $\mathrm{PG}(n, q), k \geq 3, q \geq 7$, that has more than $F_{q} \theta_{k}^{2}$ elements, is a sunflower, with

$$
F_{q}=\frac{1}{2}\left(\frac{B_{q}}{c_{q}^{2}}-\frac{1}{q}-\sqrt{\left(\frac{1}{q}-\frac{B_{q}}{c_{q}^{2}}\right)^{2}-4 B_{q}\left(\frac{1}{c_{q}^{2}}-1\right)}\right)
$$


and

$$
\begin{aligned}
B_{q} & =\left(1-c_{q}\right)^{2}\left(1-c_{q}-\frac{1}{q^{3}}\right)^{2}\left(1-c_{q}-\frac{c_{q}}{q}\right)\left(1-c_{q}-\frac{1+c_{q}}{q}\right) q, \\
c_{q} & =1-\frac{1}{\sqrt[6]{q}}-\frac{1}{2 \sqrt[3]{q}} .
\end{aligned}
$$

In particular, we have that a $(k+1,1)$-SCID in $\mathrm{PG}(n, q)$, with more than $\left(\frac{2}{\sqrt[6]{q}}+\frac{4}{\sqrt[3]{q}}-\frac{5}{\sqrt{q}}\right) \theta_{k}^{2}$ elements is a sunflower.

Proof. From Corollary 3.2, Lemma 3.4 and Lemma 3.5, we know that $F_{q} \theta_{k}^{2}$ gives an upper bound on the size $|\mathcal{S}|=(1-s) \theta_{k}^{2}$ of a $(k+1,1)$-SCID, with $\mathcal{S}$ not a sunflower. Hence, a $(k+1,1)$-SCID with more than $F_{q} \theta_{k}^{2}$ elements is a sunflower. We have to prove that

$$
\begin{aligned}
F_{q} & \leq \frac{2}{\sqrt[6]{q}}+\frac{4}{\sqrt[3]{q}-\frac{5}{\sqrt{q}}} \\
\Leftrightarrow & \frac{B_{q}}{c_{q}^{2}}-\frac{1}{q}-\sqrt{\left(\frac{1}{q}-\frac{B_{q}}{c_{q}^{2}}\right)^{2}-4 B_{q}\left(\frac{1}{c_{q}^{2}}-1\right)} \leq \frac{4}{\sqrt[6]{q}}+\frac{8}{\sqrt[3]{q}}-\frac{10}{\sqrt{q}}
\end{aligned}
$$

If $\frac{B_{q}}{c_{q}^{2}}-\frac{1}{q}-\frac{4}{\sqrt[6]{q}}-\frac{8}{\sqrt[3]{q}}+\frac{10}{\sqrt{q}} \leq 0$, then this is true for all values of $q \geq 7$. If $\frac{B_{q}}{c_{q}^{2}}-\frac{1}{q}-\frac{4}{\sqrt[6]{q}}-\frac{8}{\sqrt[3]{q}}+\frac{10}{\sqrt{q}}>0$, then it is equivalent to proving that

$$
\begin{array}{cc} 
& \left(\frac{1}{q}-\frac{B_{q}}{c_{q}^{2}}\right)^{2}-4 B_{q}\left(\frac{1}{c_{q}^{2}}-1\right) \\
\geq & \left(\frac{4}{\sqrt[6]{q}}+\frac{8}{\sqrt[3]{q}}-\frac{10}{\sqrt{q}}\right)^{2}+2\left(\frac{4}{\sqrt[6]{q}}+\frac{8}{\sqrt[3]{q}}-\frac{10}{\sqrt{q}}\right)\left(\frac{1}{q}-\frac{B_{q}}{c_{q}^{2}}\right) \\
& +\left(\frac{1}{q}-\frac{B_{q}}{c_{q}^{2}}\right)^{2} \\
\Leftrightarrow \quad & B_{q}\left(-\frac{1}{c_{q}^{2}}+1+\frac{1}{c_{q}^{2}}\left(\frac{2}{\sqrt[6]{q}}+\frac{4}{\sqrt[3]{q}}-\frac{5}{\sqrt{q}}\right)\right) \\
& \geq\left(\frac{2}{\sqrt[6]{q}}+\frac{4}{\sqrt[3]{q}}-\frac{5}{\sqrt{q}}\right)^{2}+\frac{1}{q}\left(\frac{2}{\sqrt[6]{q}}+\frac{4}{\sqrt[3]{q}}-\frac{5}{\sqrt{q}}\right) \\
\Leftrightarrow \quad & B_{q}\left(c_{q}^{2}-1+\frac{2}{\sqrt[6]{q}}+\frac{4}{\sqrt[3]{q}}-\frac{5}{\sqrt{q}}\right) \\
\geq & c_{q}^{2}\left(\left(\frac{2}{\sqrt[6]{q}}+\frac{4}{\sqrt[3]{q}}-\frac{5}{\sqrt{q}}\right)^{2}+\frac{1}{q}\left(\frac{2}{\sqrt[6]{q}}+\frac{4}{\sqrt[3]{q}}-\frac{5}{\sqrt{q}}\right)\right) \\
\stackrel{t=\sqrt[6]{q}}{\Longleftrightarrow} \quad B_{t^{6}} & \left(\frac{1}{4 t^{4}}+\frac{4}{t^{2}}-\frac{4}{t^{3}}\right) \\
& \geq\left(1-\frac{1}{t}-\frac{1}{2 t^{2}}\right)^{2}\left(\left(\frac{2}{t}+\frac{4}{t^{2}}-\frac{5}{t^{3}}\right)^{2}+\frac{1}{t^{6}}\left(\frac{2}{t}+\frac{4}{t^{2}}-\frac{5}{t^{3}}\right)\right) .
\end{array}
$$


Due to Lemma 3.3(3), it is sufficient to prove that

$$
\begin{gathered}
\left(1+\frac{1}{2 t}\right)^{2}\left(1+\frac{1}{2 t}-\frac{1}{t^{4}}\right)^{2}\left(1+\frac{1}{2 t}-\frac{1}{t^{5}}\right)\left(1+\frac{1}{2 t}-\frac{2}{t^{5}}\right)\left(\frac{1}{4 t^{4}}+\frac{4}{t^{2}}-\frac{4}{t^{3}}\right) \\
\geq\left(1-\frac{1}{t}-\frac{1}{2 t^{2}}\right)^{2}\left(\left(\frac{2}{t}+\frac{4}{t^{2}}-\frac{5}{t^{3}}\right)^{2}+\frac{1}{t^{6}}\left(\frac{2}{t}+\frac{4}{t^{2}}-\frac{5}{t^{3}}\right)\right)
\end{gathered}
$$

equivalently that

$$
\begin{aligned}
\frac{157}{4 t^{4}}+\frac{95}{4 t^{5}}-\frac{2165}{16 t^{6}}+\frac{173}{8 t^{7}}+\frac{1411}{64 t^{8}}+\frac{383}{64 t^{9}}+\frac{1313}{256 t^{10}} \\
+\frac{69}{2 t^{11}}+\frac{1177}{32 t^{12}}-\frac{37}{8 t^{13}}-\frac{3315}{128 t^{14}}-\frac{219}{8 t^{15}}-\frac{1631}{64 t^{16}}+\frac{3}{32 t^{17}} \\
\quad+\frac{557}{32 t^{18}}+\frac{151}{16 t^{19}}+\frac{293}{32 t^{20}}-\frac{1}{8 t^{21}}-\frac{11}{2 t^{22}}-\frac{3}{2 t^{23}}+\frac{1}{8 t^{24}} \geq 0 .
\end{aligned}
$$

This inequality is true for all $t \geq \sqrt[6]{7}$, and so for $q \geq 7$. So, a $(k+1,1)$ SCID in $\operatorname{PG}(n, q)$, with at least $\left(\frac{2}{\sqrt[6]{q}}+\frac{4}{\sqrt[3]{q}}-\frac{5}{\sqrt{q}}\right) \theta_{k}^{2}$ elements, has more than $F_{q} \theta_{k}^{2}$ elements. This implies that this SCID is a sunflower, which proves the theorem.

Note that the bound $1-s \leq \frac{2}{\sqrt[6]{q}}+\frac{4}{\sqrt[3]{q}}-\frac{5}{\sqrt{q}}$ only gives an improvement for the sunflower bound for $q \geq 473$, and so, it is useful for large values of $q$. For $q \geq 473$, this bound is also an improvement on the bound in [2]. For fixed, smaller values of $q$ an improved sunflower bound can be found by investigating the bound $1-s \leq F_{q}$. This bound gives an improvement on the sunflower bound if $F_{q}<1-\frac{1}{\theta_{k}}+\frac{1}{\theta_{k}^{2}}$. For $k=3$ and $k=4$, this is the case for $q \geq 9$ and $q \geq 8$ respectively. For $k>4$ we have that $F_{q}<1-\frac{1}{\theta_{k}}+\frac{1}{\theta_{k}^{2}}$, if $F_{q}<1-\frac{1}{\theta_{5}}$, which is the case for $q \geq 7$. For these values of $q$ and $k$, the bound $1-s \leq F_{q}$ improves the bound in [2].

\begin{tabular}{|c|c|c|}
\hline$q$ & $F_{q}$ & $\frac{2}{\sqrt[6]{q}}+\frac{4}{\sqrt[3]{q}}-\frac{5}{\sqrt{q}}$ \\
\hline $2^{4}$ & 0.97698136 & 1.59732210 \\
$2^{6}$ & 0.89046942 & 1.37500000 \\
$2^{8}$ & 0.78319928 & 1.11116105 \\
$2^{10}$ & 0.67282525 & 0.87056078 \\
$2^{12}$ & 0.56493296 & 0.67187500 \\
$2^{14}$ & 0.46301281 & 0.51527789 \\
$2^{16}$ & 0.37118406 & 0.39466158 \\
$2^{18}$ & 0.29280283 & 0.30273438 \\
$2^{20}$ & 0.22886576 & 0.23291485 \\
\hline
\end{tabular}

Table 1: Upper bound $F_{q}$ and $\frac{2}{\sqrt[6]{q}}+\frac{4}{\sqrt[3]{q}}-\frac{5}{\sqrt{q}}$ on $(1-s)$ for specific values of $q$.

In Table 1 we give the values of the upper bound $F_{q}$ and $\frac{2}{\sqrt[6]{q}}+\frac{4}{\sqrt[3]{q}}-\frac{5}{\sqrt{q}}$ 
on $(1-s)$, for some specific values $q$. The values in this table confirm that the bound $\frac{2}{\sqrt[6]{q}}+\frac{4}{\sqrt[3]{q}}-\frac{5}{\sqrt{q}}$ is a good approximation for $F_{q}$ for large values of $q$.

Note that for fixed values of $k$ and $q$ there is a possibility to find a slightly better bound than the bound $F_{q}$, by using our techniques. Given the fixed values for $k$ and $q$ in inequality (2), we can choose the values of $c$ and $d$ such that we get the optimal bound for $1-s$. We describe this technique in the example below.

Example 3.7. Suppose that $q=2^{8}=256$ and $k=5$, then we find from (2), that

$$
\begin{aligned}
& \left(1-\frac{s}{c d}\right)(1-d)(1-c)\left(1-c-\frac{1}{\theta_{5}}\right)^{2}\left(1-d-\frac{d}{2^{8}}\right)\left(1-d-\frac{1+d}{2^{8}}\right) 2^{8} \\
& \leq(1-s)^{2}+\frac{1-s}{2^{24}} \\
& \Leftrightarrow\left(1-\frac{s}{c d}\right) B(c, d) \leq(1-s)^{2}+\frac{1-s}{2^{24}} \\
& \Leftrightarrow(1-s)^{2}+(1-s)\left(\frac{1}{2^{24}}-\frac{B(c, d)}{c d}\right)-\left(1-\frac{1}{c d}\right) B(c, d) \geq 0 \\
& \Leftrightarrow(1-s) \leq \frac{1}{2}\left(\frac{B(c, d)}{c d}-\frac{1}{2^{24}}-\sqrt{\left(\frac{1}{2^{24}}-\frac{B(c, d)}{c d}\right)^{2}-4\left(\frac{1}{c d}-1\right) B(c, d)}\right) \\
& \text { with } B(c, d)=(1-d)(1-c)\left(1-c-\frac{1}{\theta_{5}\left(2^{8}\right)}\right)^{2}\left(1-d-\frac{d}{2^{8}}\right)\left(1-d-\frac{1+d}{2^{8}}\right) 2^{8} .
\end{aligned}
$$
By using a computer algebra package, we find a very good bound on $1-s$ for $c=0.53152285$ and $d=0.5294$. For these values we find the bound $1-s \leq 0.7825095$. Hence this gives a small improvement on the bound $1-s \leq$ $F_{q}=0.78319928$, for which we used $c\left(2^{8}\right)=d\left(2^{8}\right)=0.5244047$. Note that the bound, given by the Sunflower Theorem 1.1, and the bound given in [2] are both larger than $0.99999999 \theta_{k}^{2}$ for $q=2^{8}=256$ and $k=5$. This indicates that our new bound is a clear improvement.

\section{Acknowledgements}

The research of Jozefien D'haeseleer is supported by the FWO (Research Foundation Flanders). We would like to thank our colleague Lins Denaux for proofreading this article in detail.

\section{References}

[1] R.D. Barrolleta, E. Suárez-Canedo, L. Storme, P. Vandendriessche, On primitive constant dimension codes and a geometrical sunflower bound. Adv. Math. Commun. 11(4) : 757 - 765, (2017). 
[2] D. Bartoli, A.-E. Riet, L. Storme, P. Vandendriessche, Improvement to the sunflower bound for two classes of equidistant constant dimension subspace codes. (preprint, 2020+).

[3] A. Beutelspacher, J. Eisfeld, J. Müller, On sets of planes in projective spaces intersecting mutually in one point. Geom. Dedicata, 78(2) : 143-159, (1999).

[4] J. Eisfeld, On sets of $n$-dimensional subspaces of projective spaces intersecting mutually in an (n-2)-dimensional subspace. Discrete Math. 255 : 81-85, (2002).

[5] T. Etzion, N. Raviv, Equidistant codes in the Grassmannian. Discrete Appl. Math. $186: 87-97,(2015)$. 